\title{
SOLUBLE GROUPS WITH FEW ORBITS UNDER AUTOMORPHISMS
}

\author{
RAIMUNDO BASTOS, ALEX C. DANTAS, AND EMERSON DE MELO
}

\begin{abstract}
Let $G$ be a group. The orbits of the natural action of $\operatorname{Aut}(G)$ on $G$ are called "automorphism orbits" of $G$, and the number of automorphism orbits of $G$ is denoted by $\omega(G)$. We prove that if $G$ is a soluble group with finite rank such that $\omega(G)<\infty$, then $G$ contains a torsion-free characteristic nilpotent subgroup $K$ such that $G=K \rtimes H$, where $H$ is a finite group. Moreover, we classify the mixed order soluble groups of finite rank such that $\omega(G)=3$.
\end{abstract}

\section{INTRODUCTION}

Let $G$ be a group. The orbits of the natural action of $\operatorname{Aut}(G)$ on $G$ are called "automorphism orbits" of $G$, and the number of automorphism orbits of $G$ is denoted by $\omega(G)$. It is interesting to ask what can we say about " $G$ " only knowing $\omega(G)$. It is obvious that $\omega(G)=1$ if and only if $G=\{1\}$, and it is well known that if $G$ is a finite group then $\omega(G)=2$ if and only if $G$ is elementary abelian. In [3], T. J. Laffey and D. MacHale proved that if $G$ is a finite non-soluble group with $\omega(G) \leqslant 4$, then $G$ is isomorphic to $\operatorname{PSL}\left(2, \mathbb{F}_{4}\right)$. Later, M. Stroppel, in [8], has shown that the only finite non-abelian simple groups $G$ with $\omega(G) \leq 5$ are the groups $\operatorname{PSL}\left(2, \mathbb{F}_{q}\right)$ with $q \in\{4,7,8,9\}$. In [2], the authors prove that if $G$ is a finite non-soluble group with $\omega(G) \leq 6$, then $G$ is isomorphic to one of $\operatorname{PSL}\left(2, \mathbb{F}_{q}\right)$ with $q \in\{4,7,8,9\}, \operatorname{PSL}\left(3, \mathbb{F}_{4}\right)$ or $\operatorname{ASL}\left(2, \mathbb{F}_{4}\right)$ (answering a question of M. Stroppel, cf. [8, Problem 2.5]).

Some aspects of automorphism orbits are also investigated for infinite groups. M. Schwachhöfer and M. Stroppel in [7, Lemma 1.1], have shown that if $G$ is an abelian group with finitely many automorphism orbits, then $G=\operatorname{Tor}(G) \oplus D$, where $D$ is a characteristic torsion-free divisible subgroup of $G$ and $\operatorname{Tor}(G)$ is the set of all torsion elements in $G$. In [1, Theorem A], the authors proved that if $G$ is a FC-group with finitely many automorphism orbits, then the derived subgroup $G^{\prime}$

2010 Mathematics Subject Classification. 20E22; 20 E36.

Key words and phrases. Extensions; Automorphisms; Soluble groups.

This work was partially supported by FAPDF - Brazil. 
is finite and $G$ admits a decomposition $G=\operatorname{Tor}(G) \times A$, where $A$ is a divisible characteristic subgroup of $\mathrm{Z}(G)$. For more details concerning automorphism orbits of groups see [8].

If $G$ is a group and $r$ is a positive integer, then $G$ is said to have finite rank $r$ if each finitely generated subgroup of $G$ can be generated by $r$ or fewer elements and if $r$ is the least such integer. The next result can be viewed as a generalization of the above mentioned results from [1] and [7].

Theorem A. Let $G$ be a soluble group of finite rank. If $\omega(G)<\infty$, then $G$ has a torsion-free radicable nilpotent subgroup $K$ such that $G=$ $K \rtimes H$, where $H$ is a finite subgroup.

We do not know whether the hypothesis that $G$ has finite rank is really needed in Theorem A. The proof that we present here uses this assumption in a very essential way.

In [3], T. J. Laffey and D. MacHale showed that $G$ is a finite group in which the order $|G|$ is not prime power and $\omega(G)=3$ if and only if $|G|=p q^{n}$, the Sylow $q$-subgroup $Q$ is a normal elementary abelian subgroup of $G$ and $P$ is a Sylow $p$-subgroup which acts fixed-pointfreely on $Q$. See also [5] for groups with $\omega(G) \leqslant 3$ (almost homogeneous groups).

Recall that a group $G$ has mixed order if it contains non-trivial elements of finite order and also elements of infinite order. We obtain the following classification.

Theorem B. Let $G$ be a mixed order soluble group with finite rank. We have $\omega(G)=3$ if and only if $G=A \rtimes H$ where $|H|=p$ for some prime $p, H$ acts fixed-point-freely on $A$ and $A=\mathbb{Q}^{n}$ for some positive integer $n$.

\section{Proofs}

A well-known result in the context of extensions of finite groups, due to I. Schur, states that if $G$ is a finite group and $N$ is a normal abelian subgroup with $(|N|,|G: N|)=1$, then there exists a complement $K$ of $N$. Recall that $K$ is a complement of (a normal subgroup) $N$ in $G$ if $N \cap K=1$ and $G=N K$. In particular, $G=N \rtimes K$. Now, we prove that Schur's theorem holds under a more general assumption that $G$ contains a divisible abelian subgroup of finite index. The proof presented here is adapted from the ideas of the finite case (cf. [6, 9.1.2]).

LEMMA 2.1. Let $A$ be a divisible normal abelian subgroup of finite index of a group $G$. Then there exists a subgroup $H$ of $G$ such that $G=A \rtimes H$. 
Proof. Let $B=G / A$. From each coset $x$ in $B$ we choose a representative $t_{x}$, so that the set $T=\left\{t_{x} \mid x \in B\right\}$ is a transversal to $A$ in $G$. Since $t_{x} t_{y} A=t_{x y} A$, there is an element $c(x, y)$ of $A$ such that $t_{x} t_{y}=t_{x y} c(x, y)$. Then

$$
\begin{gathered}
\left(t_{x} t_{y}\right) t_{z}=t_{x y} c(x, y) t_{z}=t_{x y} t_{z} c(x, y)^{z}=t_{x y z} c(x y, z) c(x, y)^{z} \\
t_{x}\left(t_{y} t_{z}\right)=t_{x} t_{y z} c(y, z)=t_{x y z} c(x, y z) c(y, z)
\end{gathered}
$$

and $c(x y, z) c(x, y)^{z}=c(x, y z) c(y, z)$, for each $x, y \in B$. Consider the element $d(y)=\prod_{x \in B} c(x, y) \in A$. As $A$ is an abelian group $d(z) d(y)^{z}=d(y z) c(y, z)^{n}$, where $n=|B|$. We obtain that $d(y z)=$ $d(y)^{z} d(z) c(y, z)^{-n}$. Now, since $A$ is a divisible group, there exists $e(y) \in A$ such that $e(y)^{n}=d(y)^{-1}$ for each $y \in B$. Hence

$$
e(z y)^{-n}=\left(e(y)^{z} e(z) c(y, z)\right)^{-n} .
$$

Since $A$ is torsion-free, it follows that $e(z y)=\left(e(y)^{z} e(z) c(y, z)\right)$. Define $s_{x}=t_{x} e(x)$, then

$$
s_{y} s_{z}=t_{y} t_{z} e(y)^{z} e(z)=t_{y z} c(y, z) e(y)^{z} e(z)=t_{y z} e(y z)=s_{y z} .
$$

Thus $x \mapsto s_{x}$ defines a homomorphism $\phi: B \rightarrow G$. Now $s_{x}=1$ implies that $t_{x} \in A$ and $x=A=1_{B}$. From this we conclude that $H=B^{\phi}$ is the desired complement.

Now, we consider groups with finitely many automorphism orbits with a characteristic torsion-free soluble subgroup of finite index (see also Schur-Zassenhaus Theorem [6, 9.1.2]).

LEMma 2.2. Let $n$ be a positive integer. Let $G$ be a group such that $\omega(G)<\infty$ and $A$ a torsion-free characteristic subgroup of $G$ with finite index $n$. If $A$ is soluble, then there exists a subgroup $H$ of $G$ such that $G=A \rtimes H$.

Proof. If $A$ is abelian, then the result is immediate by Lemma 2.1. Assume that $A$ is non-abelian. Set $d$ the derived length of $A$. First we prove that $A / A^{(d-1)}$ is torsion-free. The subgroup $A^{(d-1)}$ is a torsionfree abelian divisible subgroup since $A$ is torsion-free and has finitely many automorphism orbits. If $A / A^{(d-1)}$ is not torsion-free, then we can find an element $a \in A$ such that $\left\langle a, A^{(d-1)}\right\rangle$ has a torsion-free divisible group of finite index. Then by Lemma 2.1 the subgroup $\left\langle a, A^{(d-1)}\right\rangle$ has elements of finite order. That is a contradiction. Thus $A / A^{(d-1)}$ is torsion-free.

Now, we complete the proof arguing by induction on the derived length of $A$. Consider the quotient group $\bar{G}=G / A^{(d-1)}$. By induction we deduce that there exists a finite subgroup $\bar{B}$ of order $n$ in $\bar{G}$ such that $\bar{G}=\bar{A} \rtimes \bar{B}$. Set $B$ the inverse image of $\bar{B}$. Clearly $A^{(d-1)} \leq B$ 
and $A^{(d-1)}$ has finite index $n$ in $B$. Therefore, by Lemma $2.1 B$ has a subgroup $H$ of order $n$ and so such a subgroup is a complement of $A$ in $G$. The result follows.

The following lemma is well-known. We supply the proof for the reader's convenience.

Lemma 2.3. Let $G$ be an abelian group of finite rank. If $\omega(G)<\infty$, then the torsion subgroup $\operatorname{Tor}(G)$ is finite.

Proof. Since $G$ has finitely many automorphism orbits, it follows that the exponent $\exp (\operatorname{Tor}(G))$ is bounded. As $G$ has finite rank we have that $\operatorname{Tor}(G)$ is finitely generated. We deduce that $\operatorname{Tor}(G)$ is finite, which completes the proof.

The following result provides a description of radicable nilpotent groups of finite rank (see [4, Theorem 5.3.6] for more details). Recall that a group $G$ is said to be radicable if each element is an $n$th power for every positive integer $n$.

Lemma 2.4. Let $G$ be a soluble group with finite rank. Then the following are equivalent:

(i) $G$ has no proper subgroups of finite index;

(ii) $G=G^{m}$ for all $m>0$;

(iii) $G$ is radicable and nilpotent.

We are now in a position to prove Theorem A.

Proof of Theorem $A$. We argue by induction on derived length of $G$.

Assume that $G$ is abelian. By Schwachhöfer-Stroppel's result [7], $G=D \oplus T$, where $D$ is characteristic torsion free divisible subgroup and $T$ is the torsion subgroup of $G$. By Lemma 2.3 , the torsion subgroup $T=\operatorname{Tor}(G)$ is a finite subgroup of $G$, the result follows.

Now, we assume that $G$ is non-abelian. Set $d$ the derived length of $G$. Arguing as in the previous paragraph, we deduce that $G^{(d-1)}=D_{1} \oplus T_{1}$, where $D_{1}$ is a characteristic torsion-free divisible subgroup and $T_{1}$ is the torsion subgroup of $G^{(d-1)}$ and so, $T_{1}$ is finite. By induction $G / G^{(d-1)}$ has the desired decomposition. More precisely, $G^{(d-1)}=D_{1} \oplus T_{1}$ and $G / G^{(d-1)}=\bar{A} \rtimes \bar{B}$ where $\bar{A}$ is torsion-free and $\bar{B}$ is finite. Note that $\bar{A}^{n}=\bar{A}$ for any positive integer $n$, since the quotient groups $\bar{A}^{(i)} / \bar{A}^{(i+1)}$ are torsion-free divisible groups (we can use Lemma 2.2 to conclude that each quotient is torsion-free).

Note that the centralizer $C_{G}\left(T_{1}\right)$ is a subgroup of finite index in $G$, because $G / C_{G}\left(T_{1}\right)$ embeds in the automorphism group of $T_{1}$ which has finite order. Let $A$ be the inverse image of $\bar{A}$. As $\bar{A}$ is torsion-free 
and $\bar{A}^{n}=\bar{A}$ for any positive integer $n$, we have $A \leqslant C_{G}\left(T_{1}\right)$. Thus $T_{1} \leq Z(A)$ and $\operatorname{Tor}(A)=T_{1}$. Set $K=A^{e}$, where $e=\exp \left(T_{1}\right)$. Then $K$ is torsion-free and has finite index in $G$. Therefore, by Lemma 2.2 , there exists a finite subgroup $H$ such that $G=K \rtimes H$. According to Lemma 2.4, we deduce that $K$ is a radicable nilpotent group (the subgroup $K$ has no proper subgroups of finite index). The proof is complete.

Now we will deal with Theorem B: Let Let $G$ be a mixed order soluble group with finite rank. We have $\omega(G)=3$ if and only if $G=A \rtimes H$ where $A=\mathbb{Q}^{n}$ for some positive integer $n,|H|=p$ for some prime $p$ and $H$ acts fixed-point-freely on $A$.

Proof of Theorem B. First assume that $G$ is a mixed order soluble group of finite rank and have $\omega(G)=3$. By Theorem A, $G=A \rtimes H$ where $A$ is a torsion-free radicable nilpotent subgroup and $H$ is a finite group. Since $\omega(G)=3$ and $G$ has mixed order, it follows that $A$ must be abelian (so that $A=\mathbb{Q}^{n}$ ) and $H$ is an elementary abelian $p$ subgroup. On the other hand, since $A$ is characteristic, we deduce that all elements in $G \backslash A$ have order $p$ and then $H$ acts fixed-point-freely on $A$, so that $H$ is cyclic [5, Lemma 2.2].

Conversely, suppose that $G=A \rtimes H$ where $A=\mathbb{Q}^{n},|H|=p$ for some prime $p$, and $H$ acts fixed-point-freely on $A$. First we prove that all elements in $G \backslash A$ have order $p$. Set $h \in G$ such that $H=\langle h\rangle$. Since $h^{p}=1$, we conclude that the minimal polynomials $m_{h}=m_{h}(x)$ of $T_{h}: \mathbb{Q}^{n} \rightarrow \mathbb{Q}^{n}$, given by $a \mapsto a^{h}$, divides

$$
x^{p}-1=(x-1)\left(x^{p-1}+x^{p-2}+\ldots+x+1\right) .
$$

Consequently, $m_{h}(x)=x^{p-1}+x^{p-2}+\ldots+x+1$, because $h$ acts fixedpoint-freely on $A$. Thus using the identity $(x y)^{n}=x^{n}\left(y^{x^{n-1}}\right) \ldots y^{x} y$, we obtain

$$
\left(h^{j} a\right)^{p}=h^{j p}\left(a a^{h^{j}} \ldots a^{h^{(p-1) j}}\right)=1
$$

for all $a \in \mathbb{Q}^{n}$. So all elements of $G \backslash A$ have order exactly $p$ and act fixed-point-freely on $A$.

Now, let $b, c \in A \backslash\{1\}$ and $\alpha, \beta \in G \backslash A$. By Cyclic Decomposition Theorem, there exist $b_{1}, b_{2}, \ldots, b_{t}, c_{1}, c_{2}, \ldots, c_{t} \in A$ such that

$$
\left\{b_{1}, b_{1}^{\alpha}, \ldots, b_{1}^{\alpha^{p-2}}, \ldots, b_{t}, b_{t}^{\alpha}, \ldots, b_{t}^{\alpha^{p-2}}\right\}
$$

and

$$
\left\{c_{1}, c_{1}^{\beta}, \ldots, c_{1}^{\beta^{p-2}}, \ldots, c_{t}, c_{t}^{\beta}, \ldots, c_{t}^{\beta^{p-2}}\right\}
$$


are bases of $A$. Without loss of generality we can assume that $b=b_{1}$ and $c=c_{1}$. Thus the map given by

$$
b_{i} \mapsto c_{i} \text { and } \alpha \mapsto \beta,
$$

where $i=1, \ldots, t$ extends to an automorphism of $G$. Hence all nontrivial elements of $A$ belong to the same orbit under the action of $\operatorname{Aut}(G)$, and all elements in $G \backslash A$ are in the same orbit under the action of $\operatorname{Aut}(G)$. The proof is complete.

\section{REFERENCES}

[1] R. Bastos and A. C. Dantas, FC-groups with finitely many automorphism orbits, J. Algebra, 516 (2018) pp. 401-413.

[2] R. Bastos, A. C. Dantas and M. Garonzi, Finite groups with six or seven automorphism orbits, J. Group Theory, 21 (2017) pp. 945-954.

[3] T. J. Laffey and D. MacHale, Automorphism orbits of finite groups, J. Austral. Math. Soc. Ser. A, 40(2) (1986) pp. 253-260.

[4] J. C. Lennox and D. J.S. Robinson, The Theory of Infinite Soluble Groups, Clarendon Press, Oxford, 2004.

[5] H. Mäurer and M. Stroppel, Groups that are almost homogeneous, Geom. Dedicata, 68 (1997) pp. 229-243.

[6] D. J.S. Robinson, A course in the theory of groups, 2nd edition, SpringerVerlag, New York, 1996.

[7] M. Schwachhöfer and M. Stroppel, Finding representatives for the orbits under the automorphism group of a bounded abelian group, J. Algebra, 211 (1999) pp. 225-239.

[8] M. Stroppel, Locally compact groups with few orbits under automorphisms, Top. Proc., 26(2) (2002) pp. 819-842.

Departamento de Matemática, Universidade de Brasília, BrasiliaDF, 70910-900 BRAZIL

E-mail address: (Bastos) bastos@mat.unb.br

E-mail address: (Dantas) alexcdan@gmail.br

E-mail address: (de Melo) emersonueg@hotmail.com 